Proyecciones

Vol. 28, No 1, pp.89-109, May 2009.

Universidad Católica del Norte

Antofagasta - Chile

\title{
AN ABSTRACT GLIDING HUMP PROPERTY
}

\author{
CHARLES SWARTZ \\ NEW MEXICO STATE UNIVERSITY, U. S. A. \\ Received: December 2008. Accepted: March 2009
}

\begin{abstract}
In this paper we introduce an abstract gliding hump property for sequence spaces which includes the signed weak and strong gliding hump properties as special cases. Further examples of sequence spaces satisfying the abstract gliding hump property are given, We then derive results concerning uniform convergence in $\beta$-duals, Hahn-Schur theorems and Orlicz-Pettis theorems for multiplier convergent series whose multiplier space satisfies the abstract gliding hump property.
\end{abstract}


1.

Gliding hump properties have been used to treat a number of topics in sequence spaces such as weak sequential completeness of $\beta$-duals ([No], [St]), uniform convergence of elements in $\beta$-duals ([StSw1]), uniform boundedness principles ([Sw4]), Orlicz-Pettis theorems for multiplier convergent series ([StSw2], [Sw5]) and Hahn-Schur theorems for multiplier convergent series ([Sw6]). In this note we introduce an abstract gliding hump property which include the signed weak gliding hump property and the signed strong gliding hump property as well as new gliding hump properties. We then derive general results concerning uniform convergence in $\beta$-duals, Hahn-Schur theorems, sequential completeness of $\beta$-duals and Orlicz-Pettis theorems which contain many known results as special cases. The general abstract result includes a number of known results for multiplier spaces with the signed weak gliding hump property and the signed strong gliding hump property. One of the special cases of the gliding hump property leads to a number of new results pertaining to uniform convergence over compact subsets of multipliers for multiplier convergent series while another special case gives uniform convergence over order bounded intervals in the multiplier space.

Throughout this note $X, Y$ will denote (Hausdorff) topological vector spaces (assumed real for convenience) and $L(X, Y)$ will denote the space of continuous linear operators from $X$ into $Y$. If $A$ is a family of bounded subsets of $X, L_{\text {cal } A}(X, Y)$ will denote $L(X, Y)$ with the topology of uniform convergence on the elements of calA. If calA is the family of all finite (bounded, compact) subsets of $X$, the topology of uniform convergence on cal $A$ will be denoted by $L_{s}(X, Y)\left(L_{b}(X, Y), L_{c}(X, Y)\right)$. Further, $E$ will be a vector space of $X$ valued sequences which contains the space $c_{00}(X)$ of all $X$ valued sequences which are eventually 0 . If $x \in E$, the $j^{\text {th }}$ coordinate of $x$ will be denoted by $x_{j}$ so $x=\left\{x_{j}\right\}$. The $\beta$-dual of $E$ with respect to $Y$ is defined to be

$E^{\beta Y}=\left\{\left\{T_{j}\right\}: T_{j} \in L(X, Y), \sum_{j=1}^{\infty} T_{j} x_{j}\right.$ converges for every $\left.x=\left\{x_{j}\right\} \in E\right\}$.

If $T=\left\{T_{j}\right\} \in E^{\beta Y}$ and $x=\left\{x_{j}\right\} \in E$, we write $T \cdot x=\sum_{j=1}^{\infty} T_{j} x_{j}$. A series $\sum_{j} T_{j}$ in $L(X, Y)$ is $E$ multiplier convergent if the series $\sum_{j=1}^{\infty} T_{j} x_{j}$ converges for every $x=\left\{x_{j}\right\} \in E$ so the $\beta$-dual of $E$ with respect to $Y$ is just the space of all $E$ multiplier convergent series in $L(X, Y)$; the elements of $E$ are called multipliers.

If $\lambda$ is a scalar sequence space which contains $c_{00}$, the space of all 
sequences which are eventually 0 , a series $\sum_{j} x_{j}$ in $X$ is $\lambda$ multiplier convergent iff the series $\sum_{j=1}^{\infty} t_{j} x_{j}$ converges for every $t=\left\{t_{j}\right\} \in \lambda$ (here we are identifying $X$ with $L(R, X)$ in the definition above). For example, if $m_{0}$ is the scalar sequence space of all sequences with finite range, a series is $m_{0}$ multiplier convergent iff the series is subseries convergent. If $\lambda=l^{\infty}$, the $l^{\infty}$ multiplier convergent series are often called bounded multiplier convergent series

We now define our abstract gliding hump property. An interval in $N$ is a set of the form $[m, n]=\{j \in N: m \leq j \leq n\}$ and a sequence of intervals $\left\{I_{j}\right\}$ is increasing if $\max I_{j}<\min I_{j+1}$. If $I \subset N, \chi_{I}$ will denote the characteristic function of $I$ and if $x=\left\{x_{j}\right\}$ is any vector or scalar sequence $\chi_{I} x$ will denote the coordinatewise product of $\chi_{I}$ and $x$.

Throughout this note $c a l F$ will be a family of subsets of $E$.

Definition 1. The space $E$ has the signed calF gliding hump property (signed calF-GHP) if for every $F \in$ calF whenever $\left\{x^{k}\right\} \subset F$ and $\left\{I_{k}\right\}$ is an increasing sequence of intervals, there exist a sequence of signs $\left\{s_{k}\right\}$ and a subsequence $\left\{n_{k}\right\}$ such that the coordinate sum of the series $\sum_{k=1}^{\infty} s_{k} \chi_{I_{k}} x^{n_{k}} \in$ $E$. If all of the signs above can be chosen equal to 1, then $E$ has the calF gliding hump property (calF-GHP).

We give several examples.

A sequence space $E$ has the signed weak gliding property (signedWGHP) if whenever $x \in E$ and $\left\{I_{j}\right\}$ is an increasing sequence of intervals, there exist a sequence of signs $\left\{s_{j}\right\}$ and a subsequence $\left\{n_{j}\right\}$ such that the coordinate sum of the series $\sum_{j=1}^{\infty} s_{j} \chi_{I_{n_{j}}} x \in E$ ([St]); if all of the signs $\left\{s_{j}\right\}$ can be chosen equal to 1 , the space has the weak gliding hump property (WGHP) ([No]). For example, in the scalar case any monotone space such as $c_{0}$ or $l^{p}(0<p \leq \infty)$ or $\delta=\left\{\left\{t_{j}\right\}: \sup \left|t_{j}\right|^{1 / j}<\infty\right\}$ has WGHP while $b s$ has signed-WGHP but not WGHP ( for the sequence spaces being used, see Boos $([\mathrm{Bo}]))$. In the vector case, the space $c_{0}(X)$ of all $X$ valued sequences which converge to 0 has WGHP; the spaces $l^{p}(X)$ described later also have WGHP.

Example 2. If calF is the family of all finite subsets, the signed calFGHP (calF-GHP) is just the signed weak gliding hump property (weak gliding hump property).

The space $E$ is a $\mathrm{K}$-space if $E$ has a (Hausdorff) vector topology such that the coordinate mappings $x=\left\{x_{j}\right\} \rightarrow x_{j}$ from $E$ into $X$ are continuous for all $j \in N$. The K-space $E$ has the signed strong gliding hump 
property (signed-SGHP) if whenever $\left\{x^{k}\right\}$ is a bounded sequence from $E$ and $\left\{I_{k}\right\}$ is an increasing sequence of intervals, there exist a sequence of signs $\left\{s_{k}\right\}$ and a subsequence $\left\{n_{k}\right\}$ such that the coordinate sum of the series $\sum_{k=1}^{\infty} s_{k} \chi_{I_{n_{k}}} x^{n_{k}} \in E([\mathrm{Sw}])$; if all the signs can be chosen equal to $1, E$ has the strong gliding hump property (SGHP) ([No]). For example, $l^{\infty}$ and $\delta$ have SGHP and $b s$ has signed-SGHP ([Sw6]); other spaces with these properties can be constructed using the methods in [BSS]. The vector valued space $l^{\infty}(X)$ described later has SGHP.

Example 3. Assume $E$ is a $K$-space. If cal $F$ is the family of all bounded subsets of $E$, the signed calF-GHP (calF-GHP) is just the signed strong gliding hump property (strong gliding hump property).

Example 4. If $E$ equals $l^{\infty}$ or $m_{0}$ and cal $F$ is the family of all sequences of 0 's and 1's, then $E$ has calF-GHP and this corresponds to the strong gliding hump property.

We give a further important example which will lead to a number of new results and which requires some preliminary notation and results.

If $z \in X$ and $k \in N, e^{k} \otimes z$ will denote the $X$ valued sequence with $z$ in the $k^{t h}$ coordinate and 0 in the other coordinates.

Definition 5. If $E$ is a $K$-space, a subset $F \subset E$ has uniform tails if whenever $\left\{x^{k}\right\} \subset F$, then there exists $\left\{n_{k}\right\}$ such that $\lim _{N \rightarrow \infty} \sum_{j=N}^{\infty} e^{j} \otimes$ $x_{j}^{n_{k}}=0$ uniformly for $k \in N$, where $\sum_{j=N}^{\infty} e^{j} \otimes x_{j}^{n_{k}}$ is the coordinate sum of the series.

Subsets with uniform tails have been previously introduced in $c_{0}(X)$ and $l^{p}(X)(1 \leq p<\infty)$ when $X$ is a Banach space in [LWZ], [ZLY].

A quasi-norm on a vector space $X$ is a function $|\cdot|: X \rightarrow[0, \infty)$ such that $|x+y| \leq|x|+|y|,|-x|=|x|,|0|=0$; any quasi-norm induces a semi-metric $d$ on $X$ by $d(x, y)=|x-y|$. A space $X$ is a quasi-normed space if $X$ has a quasi-norm $|\cdot|$ such that $X$ is a topological vector space under the induced semi-metric.

Theorem 6. Let $(E,|\cdot|)$ be a complete, quasi-normed $K$-space. If calF is the family of all subsets of $E$ with uniform tails, then $E$ has calF-GHP.

Proof: Let $F \in c a l F,\left\{x^{k}\right\} \subset F$ and $\left\{I_{k}\right\}$ be an increasing sequence of intervals. Let $\left\{x^{n_{k}}\right\}$ be as in Definition 5. Pick an increasing sequence $\left\{k_{l}\right\}$ such that $\left|\sum_{j \in I_{k_{l}}} e^{j} \otimes x_{j}^{n_{k_{l}}}\right|<1 / 2^{l}$. Put $z^{l}=\sum_{j \in I_{k_{l}}} e^{j} \otimes x_{j}^{n_{k_{l}}}$. Then 
$\sum_{l} z^{l}$ is an absolutely convergent series and, therefore, converges to some $z \in E$. Since $E$ is a K-space, the series $\sum_{l=1}^{\infty} z_{j}=\sum_{j=1}^{\infty} \sum_{j \in I_{k_{l}}} e^{j} \otimes x_{j}^{n_{k_{l}}}$ also converges coordinatewise to $z$.

We now give an example of subsets with uniform tails.

If $\lambda$ is a scalar $A K-F K$ space, then a subset of $\lambda$ has uniform tails iff the set is relatively compact ([Sw1]10.1.15). We have a more general result for vector valued sequence spaces. The space $E$ is an AK-space if $E$ is a K-space and if $x=\sum_{k=1}^{\infty} e^{k} \otimes x_{k}$ [convergence in $E$ ] for every $x=\left\{x_{k}\right\} \in E$. A quasi-normed AK-space $(E,|\cdot|)$ has a monotone basis if $\left|\sum_{j=1}^{k} e^{j} \otimes x_{j}\right| \uparrow\left|\sum_{j=1}^{\infty} e^{j} \otimes x_{j}\right|=|x|$ for every $x \in E$. Any scalar AK-FK space can be remetrized so the quasi-norm is monotone ([Sw1]10.1.13).

Theorem 7. If the quasi-normed space $A K$-space $E$ has a monotone basis and $K \subset E$ is precompact, then $\lim _{N \rightarrow \infty} \sum_{j=N}^{\infty} e^{j} \otimes x_{j}=0$ uniformly for $x=\left\{x_{j}\right\} \in K$. In particular, $K$ has uniform tails.

Proof: Let $\epsilon>0$. Set $s_{n}(x)=\sum_{j=1}^{n} e^{j} \otimes x_{j}, R_{n}(x)=\sum_{j=n+1}^{\infty} e^{j} \otimes$ $x_{j}$ for $x \in K$. There exists a finite $\epsilon$-net , $x^{1}, \ldots, x^{p} \in K$ for $K$. Since $\lim _{n} R_{n}\left(x^{j}\right)=0$ for $j=1, \ldots, p$, there exists $N$ such that $n \geq N$ implies $\left|R_{n}\left(x^{j}\right)\right|<\epsilon$ for $j=1, \ldots, p$. If $x \in K$, there exists $j$ such that $\left|x-x^{j}\right|<\epsilon$. If $n \geq N$, then $\left|R_{n}(x)\right|=\left|x-s_{n}(x)\right| \leq\left|s_{n}\left(x^{j}\right)-s_{n}(x)\right|+\left|R_{n}\left(x^{j}\right)\right|+$ $\left|x-x^{j}\right|<3 \epsilon$.

Unlike the scalar case a subset with uniform tails may not be precompact. Consider $X$ an infinite dimensional Banach space and the set $K=\left\{\left\{x_{j}\right\}: x_{j} \in X,\left\|x_{j}\right\| \leq 1 / j, j \in N\right\}$.

From Theorems 6 and 7 , we have

Corollary 8. Let $E$ be a complete, quasi-normed AK-space with a monotone basis and calF the family of all relatively compact subsets of $E$. Then $E$ has calF-GHP.

For example, the scalar spaces $c_{0}, c s, b v_{0}$ and $l^{p}(0<p<\infty)$ all have $c a l F-G H P$, where $c a l F$ is the family of all relatively compact subsets. The same remark applies to the vector valued sequence spaces $c_{0}(X)$ and $l^{p}(X)$ $(0<p<\infty)$, when $X$ is a complete quasi-normed space $\left(c_{0}(X)\right.$ is the space of all $X$ valued null sequences with the quasi-norm $\left|\left\{x_{j}\right\}\right|_{\infty}=\sup \left|x_{j}\right|$ and for $1 \leq p<\infty, l^{p}(X)$ is the space of all $X$ valued sequences with the quasinorm $\left|\left\{x_{j}\right\}\right|_{p}=\left(\sum_{j=1}^{\infty}\left|x_{j}\right|^{p}\right)^{1 / p}<\infty$, for $0<p<1, l^{p}(X)$ is the space of 
all $X$ valued sequences with the quasi-norm $\left|\left\{x_{j}\right\}\right|_{p}=\sum_{j=1}^{\infty}\left|x_{j}\right|^{p}<\infty$ and $l^{\infty}(X)$ is the space of all $X$ valued sequences with $\left.\left|\left\{x_{j}\right\}\right|_{\infty}=\sup _{j}\left|x_{j}\right|<\infty\right)$.

Likewise, if $E$ is the strict inductive limit of locally convex spaces satisfying the assumptions in Corollary 8 , the family of all relatively compact subsets calF of $E$ has calF-GHP.

We give a further example in ordered sequence spaces. A (real) sequence space $\lambda$ which is ordered coordinatewise is solid (normal) if whenever $t=$ $\left\{t_{j}\right\} \in \lambda$ and $\left|s_{j}\right| \leq\left|t_{j}\right|$ for all $j$, then $s=\left\{s_{j}\right\} \in \lambda$. For example, $c_{00}, c_{0}, l^{p}$ $(0<p \leq \infty), \delta$ and $d=\left\{\left\{t_{j}\right\}: \lim \sup \left|t_{j}\right|^{1 / j}=0\right\}$ are solid whereas $c, c s, b s$ are not solid. The spaces $l^{p+}=\cap_{q>p} l^{q}$ and $l^{p-}=\cup_{q<p} l^{q}$ introduced in [MM] are also solid. If $t \in \lambda$ and $t \geq 0$, let $[-t, t]=\left\{s=\left\{s_{j}\right\} \in \lambda:\left|s_{j}\right| \leq\left|t_{j}\right|\right.$ for all $j$ \} be the symmetric interval generated by $t$.

Example 9. Let $\lambda$ be a solid sequence space. Let calF be the family of all symmetric order intervals generated by the non-negative sequences in $\lambda$. Since $\lambda$ is solid, $\lambda$ has the calF gliding hump property.

Like the weak gliding hump property, the property in the example above is algebraic and does not require a topology on the space of multipliers and so applies to spaces such as

$$
d=\left\{t=\left\{t_{j}\right\}: \sup _{j}\left|t_{j}\right|^{1 / j}<\infty\right\} \text { and } \delta=\left\{t=\left\{t_{j}\right\}: \limsup _{j}\left|t_{j}\right|^{1 / j}=0\right\}
$$

(see $[\mathrm{Bo}],[\mathrm{KG}])$.

We now proceed to establish uniform convergent results in the $\beta$-duals of spaces with calF-GHP.

Lemma 10. Let $\sum_{j} T_{j}$ be $E$ multiplier convergent and $B \subset E$. If the series $\sum_{j} T_{j} x_{j}$ do not converge uniformly for $x=\left\{x_{j}\right\} \in B$, then there exist a symmetric neighborhood $V$ of $0,\left\{x^{k}\right\} \subset B$ and an increasing sequence of intervals $\left\{I_{k}\right\}$ such that $\sum_{j \in I_{k}} T_{j} x_{j}^{k} \notin V$.

Proof: There exists a symmetric neighborhood of $0, U$, such that for every $k$ there exist $x^{k} \in B, m_{k}>k$ such that $\sum_{j=m_{k}}^{\infty} T_{j} x_{j}^{k} \notin U$. Pick a symmetric neighborhood of $0, V$, such that $V+V \subset U$. For $k=1$ let $m_{1}>1$ and $x^{1} \in B$ satisfy the condition above. There exists $n_{1}>m_{1}$ such that $\sum_{j=n_{1}+1}^{\infty} T_{j} x_{j}^{1} \in V$. Then $\sum_{j=m_{1}}^{n_{1}} T_{j} x_{j}^{1}=\sum_{j=m_{1}}^{\infty} T_{j} x_{j}^{1}-\sum_{j=n_{1}+1}^{\infty} T_{j} x_{j}^{1} \notin V$. Put $I_{1}=\left[m_{1}, n_{1}\right]$ and continue the construction.

Theorem 11. Assume that $E$ has signed calF-GHP and $\sum_{j} T_{j}$ is $E$ multiplier convergent. If $F \in$ calF, then the series $\sum_{j=1}^{\infty} T_{j} x_{j}$ converge uniformly for $x=\left\{x_{j}\right\} \in F$. 
Proof: Suppose the conclusion fails and let the notation be as in Lemma 10 so $\sum_{j \in I_{k}} T_{j} x_{j}^{k} \notin V$ with $x^{k} \in F$. By signed calF-GHP, There exist $\left\{p_{k}\right\}$ and signs $\left\{s_{k}\right\}$ such that $x=\sum_{k=1}^{\infty} s_{k} \chi_{I_{p_{k}}} x^{p_{k}} \in E$. But, then the series $\sum_{j=1}^{\infty} T_{j} x_{j}$ doesn't converge since $\sum_{j \in I_{p_{k}}} T_{j} x_{j}=s_{k} \sum_{j \in I_{p_{k}}} T_{j} x_{j}^{p_{k}} \notin V$,i.e., the series doesn't satisfy the Cauchy condition.

From Example 2 and Theorem 11 we have that if $E$ has signed-SGHP and $\sum_{j} T_{j}$ is $E$ multiplier convergent, then the series $\sum_{j=1}^{\infty} T_{j} x_{j}$ converge uniformly for $x$ belonging to bounded subsets of $E$ a result derived in [Sw6] Theorem 3.

From Theorems 6 and 11 we have uniform convergence on any set with uniform tails when $E$ is a complete quasi-normed space; this was established for $E=c_{0}(X)$ and $l^{p}(X)$ when $X$ is a Banach space in [LWZ]. Under additional hypothesis, the family of subsets with uniform tails is the largest family for which the conclusion of Theorem 11 holds,i.e., the family of subsets with uniform tails is optimal for the conclusion of Theorem 11 to hold.

Theorem 12. Let $K \subset E$ and consider the following conditions:

(i) $K \subset E$ has uniform tails

(ii) for every complete, quasi - normed space $Y$ and for every $T \in E^{\beta Y}$, the series $\sum_{j=1}^{\infty} T_{j} x_{j}$ converge uniformly for $x \in K$.

Then (i) implies (ii) if $E$ is a complete quasi-normed and (ii) implies (i) if $E$ is an $A K$-space and $X$ is quasi-normed.

Proof: The first statement follows from Theorems 6 and 11 .

Suppose $K$ does not have uniform tails and let $|\cdot|$ be a quasi-norm generating the topology of $X$. Then there exists $\epsilon>0$ such that for every $k$ there exist $x^{k} \in K, m_{k}>k$ such that $\left|\sum_{j=m_{k}}^{\infty} x_{j}^{k} \otimes e^{j}\right|>\epsilon$. For $k=1$ there exist $x^{1} \in K, m_{1}>1$ such that $\left|\sum_{j=m_{1}}^{\infty} x_{j}^{1} \otimes e^{j}\right|>\epsilon$ and there exists $n_{1}>m_{1}$ such that $\left|\sum_{j=m_{1}}^{n_{1}} x_{j}^{1} \otimes e^{j}\right|>\epsilon$. Similarly there exists $x^{2} \in K, n_{2}>$ $m_{2}>n_{1}$ such that $\left|\sum_{j=m_{2}}^{n_{2}} x_{j}^{1} \otimes e^{j}\right|>\epsilon$. Continuing produces a sequence $\left\{x^{k}\right\} \subset K$, increasing sequences $\left\{m_{k}\right\},\left\{n_{k}\right\}$ with $m_{k}<n_{k}<m_{k+1}$ with

$$
(*)\left|\sum_{j=m_{k}}^{n_{k}} x_{j}^{k} \otimes e^{j}\right|>\epsilon \text {. }
$$


Define $T_{i}: X \rightarrow E$ by $T_{i} x=x \otimes e^{i}$ so $T_{i}$ is linear and continuous. Set $T=\left\{T_{i}\right\}$. Then $T \in E^{\beta E}$ since for every $x \in E, \sum_{i=1}^{\infty} T_{i} x_{i}=\sum_{i=1}^{\infty} x_{i} \otimes e^{i}$ by the AK assumption. However, the series $\sum_{i=1}^{\infty} T_{i} x_{i}^{k}$ do not converge uniformly for $k \in N$ by $(*)$.

From Corollary 8 and Theorem 11 we have that if $E$ is a complete quasi-normed AK-space with a monotone basis and $\sum_{j} T_{j}$ is $E$ multiplier convergent, then the series $\sum_{j=1}^{\infty} T_{j} x_{j}$ converge uniformly for $x$ belonging to relatively compact subsets of $E$; this result appears to be new and applies to many spaces without the signed-SGHP such as $c_{0}, c s, b v_{0}, c_{0}(X)$ and $l^{p}(X)$ $(1 \leq p<\infty)$; see [LWZ] for the case of $c_{0}(X)$ and $l^{p}(X)$ when $X$ is a Banach space.

Likewise, if $\lambda$ is a solid sequence space and $\sum_{j} x_{j}$ is a $\lambda$ multiplier convergent series in $X$, then from Example 9 and Theorem 11 the series $\sum_{j=1}^{\infty} t_{j} x_{j}$ converge uniformly when $t$ runs over symmetric order bounded intervals in $\lambda$. This uniform convergence observation also seems to be new. If $\lambda=l^{\infty}$, then the family of order bounded symmetric intervals coincides with the family of bounded subsets of $l^{\infty}$ so Theorem 11 covers this case. However, there are numerous solid sequence spaces not covered by the previous results. For example, the spaces $d=\left\{t=\left\{t_{j}\right\}: \sup _{j}\left|t_{j}\right|^{1 / j}<\infty\right\}, \delta=\{t=$ $\left.\left\{t_{j}\right\}: \lim \sup _{j}\left|t_{j}\right|^{1 / j}=0\right\}$ and $l^{p-}=\cup_{q<p} l^{q}$ are solid ([Bo],[KG],[MM]). In particular, $l^{p-}=\cup_{q<p} l^{q}$ with the inductive limit topology from $\left\{l^{q}: q<p\right\}$ is an LB-AK space which is not metrizable and to which Corollary 8 does not apply. ([MM]).

We next consider uniform convergence over sequences of elements in $\beta$ duals. The proof of this result uses the Antosik-Mikusinski Matrix Theorem which can be found in [St],[Sw2]2.2.4.

Theorem 13. Assume that $E$ has signed calF-GHP, $T^{k} \in E^{\beta Y}$ and $F \in$ calF. If $\lim _{k} T^{k} \cdot x$ exists for every $x \in X$, then the series $\sum_{j=1}^{\infty} T_{j}^{k} x_{j}$ converge uniformly for $k \in N, x \in F$.

Proof: If the conclusion fails, there exists a neighborhood, $U$, of 0 such that for every $n$ there exist $k_{n}, x^{n} \in F$ an interval $I_{n}$ with $\min I_{n}>n$ and $\sum_{j \in I_{n}} T_{j}^{k_{n}} x_{j}^{n} \notin U$. By this condition for $n=1$ there exist $k_{1}>1$, $x^{1} \in F$, an interval $I_{1}$ with min $I_{1}>1$ and $\sum_{j \in I_{1}} T_{j}^{k_{1}} x_{j}^{1} \notin U$. By Theorem 11 there exists $m^{\prime}>\max I_{1}$ such that $\sum_{j=p}^{q} T_{j}^{k} x_{j} \in U$ for $1 \leq k \leq k_{1}$, $x \in F, q>p \geq m^{\prime}$. Again by the condition above, there exist $k_{2}, x^{2} \in F$, an interval $I_{2}$ with min $I_{2}>m^{\prime}$ and $\sum_{j \in I_{2}} T_{j}^{k_{2}} x_{j}^{2} \notin U$. Hence, $k_{2}>k_{1}$. 
Continuing this construction produces an increasing sequence $\left\{k_{i}\right\}, x^{i} \in F$, increasing intervals $\left\{I_{i}\right\}$ such that

$$
\text { (\#) } \sum_{j \in I_{i}} T_{j}^{k_{i}} x_{j}^{i} \notin U
$$

Define a matrix

$$
M=\left[m_{i j}\right]=\left[T^{k_{i}} \cdot \chi_{I_{j}} x^{j}\right] .
$$

We claim that $M$ is a signed calK matrix ([Sw2]2.2.4). First the columns of $M$ converge by hypothesis. Given an increasing sequence of integers, there exist an increasing sequence $\left\{p_{j}\right\}$ and signs $\left\{s_{j}\right\}$ such that $x=$ $\sum_{j=1}^{\infty} s_{j} \chi_{I_{p_{j}}} x^{p_{j}} \in E$. Then

$$
\sum_{j=1}^{\infty} s_{j} m_{i p_{j}}=\sum_{j=1}^{\infty} s_{j} T^{k_{i}} \cdot \chi_{I_{p_{j}}} x^{p_{j}}=T^{k_{i}} \cdot x
$$

and $\lim _{i} T^{k_{i}} \cdot x$ exists by hypothesis. Hence, $M$ is a signed calK matrix. By the signed version of the Antosik-Mikusinski Matrix Theorem, the diagonal of $M$ converges to 0 . But, this contradicts (\#).

As a special case of Theorem 13 we have that if $E$ has signed-WGHP (signed-SGHP), then the series in the conclusion of Theorem 13 converge uniformly for $k \in N$ ( $k \in N$ and $x$ belonging to bounded subsets of $E$ ); these results were derived in [StSw1] Theorem 1 (also see [Sw6] Theorem 8). From Theorem 6 the series in the conclusion of Theorem 13 holds for $k \in N$ and $x$ belonging to sets with uniform tails when $E$ is a complete, quasi-normed K-space. From Corollary 8 if $E$ is a complete quasi-normed AK-space with a monotone basis, then the series in Theorem 13 converge uniformly for $k \in N$ and $x$ belonging to relatively compact subsets of $E$; this result seems to be new and again applies to a number of spaces not covered in the examples above.

Likewise, by Example 9, if $\lambda$ is a solid sequence space, then the series $\sum_{j=1}^{\infty} t_{j} x_{j}^{k}$ in Theorem 13 converge uniformly for $t=\left\{t_{j}\right\}$ running over order bounded intervals in $\lambda$, a result that seems to be new.

We next consider an abstract version of the Hahn-Schur Theorem. The original scalar version of the theorem assets that if the series $\sum_{j} t_{i j}$ are absolutely convergent for each $i \in N$ and if $\lim _{i} \sum_{j=1}^{\infty} t_{i j} s_{j}$ exists for each $\left\{s_{j}\right\} \in l^{\infty}\left(\left\{s_{j}\right\} \in m_{0}\right)$ and if $t_{j}=\lim _{i} t_{i j}$, then $\sum_{j} t_{j}$ is absolutely convergent and $\sum_{j=1}^{\infty}\left|t_{i j}-t_{j}\right| \rightarrow 0$ ([Sw1]9.5.3, [Kö]22.4.(2)). There have been 
a number of vector versions of the theorem for multiplier convergent series given; for example, see [Sw2] Chapters 8 and 9 for such results and references. We establish two preliminary results.

Proposition 14. Assume $T^{i} \in E^{\beta Y}$, for every $x \in E$ the series $\sum_{j=1}^{\infty} T_{j}^{i} x_{j}$ converge uniformly for $i \in N$ and $\lim _{i} T^{i} \cdot x$ exists. If for each $j \in N$, there exists $T_{j} \in L(X, Y)$ such that $\lim _{i} T_{j}^{i}=T_{j}$ in the strong operator topology, then $\sum_{j} T_{j}$ is $E$ multiplier convergent and $\lim _{i} \sum_{j=1}^{\infty} T_{j}^{i} x_{j}=\sum_{j=1}^{\infty} T_{j} x_{j}$ for every $x=\left\{x_{j}\right\} \in E$.

Proof: Let $x \in E$ and put $u=\lim _{i} T^{i} \cdot x$. Let $U$ be a balanced neighborhood of 0 and pick a closed, balanced neighborhood of $0, V$, such that $V+V+V \subset U$. By hypothesis there exists $N$ such that $\sum_{j=n}^{\infty} T_{j}^{i} x_{j} \in V$ for $n \geq N, i \in N$. Fix $n \geq N$ and pick $k=k_{n}$ such that $\sum_{j=1}^{\infty} T_{j}^{k} x_{j}-u \in V$ and $\sum_{j=1}^{n}\left(T_{j}^{k}-T_{j}\right) x_{j} \in V$. Then

$\sum_{j=1}^{n} T_{j} x_{j}-u=\left(\sum_{j=1}^{\infty} T_{j}^{k} x_{j}-u\right)+\sum_{j=1}^{n}\left(T_{j}-T_{j}^{k}\right) x_{j}-\sum_{j=n+1}^{\infty} T_{j}^{k} x_{j} \in V+V+V \subset U$.

If $F \in c a l F$, set $F_{j}=\left\{x_{j}: x \in F\right\}$ and $c a l F_{j}=\left\{F_{j}: F \in c a l F\right\}$.

Theorem 15. Assume $T^{i} \in E^{\beta Y}$ for $i \in N$, for every $F \in$ calF the series $\sum_{j=1}^{\infty} T_{j}^{i} x_{j}$ converge uniformly for $i \in N, x \in F$ and $\lim _{i} T^{i} \cdot x$ exists for every $x \in E$. If for every $j$ there exists $T_{j} \in L(X, Y)$ such that $\lim _{i} T_{j}^{i}=T_{j}$ in $L_{c a l F_{j}}(X, Y)$, then $\sum_{j} T_{j}$ is $E$ multiplier convergent and $\lim _{i} \sum_{j=1}^{\infty} T_{j}^{i} x_{j}=$ $\sum_{j=1}^{\infty} T_{j} x_{j}$ uniformly for $x$ belonging to any $F \in$ cal $F$.

Proof: If $x \in E, \lim _{i} \sum_{j=1}^{\infty} T_{j}^{i} x_{j}=\sum_{j=1}^{\infty} t_{j} x_{j}$ by Proposition 14. Let $F \in$ calF. Let $U$ be a neighborhood of 0 and pick a closed, balanced neighborhood of $0, V$, such that $V+V+V \subset U$. There exists $M$ such that $n \geq M$ implies $\sum_{j=n}^{\infty} T_{j}^{i} x_{j} \in V$ for $i \in N, x \in F$. By the first observation above $\sum_{j=n}^{\infty} T_{j} x_{j} \in V$ for $n \geq M, x \in F$. Since $F_{j} \in$ calF $_{j}$ for $1 \leq j \leq M$, there exists $q \geq M$ such that $\sum_{j=1}^{M}\left(T_{j}^{i}-T_{j}\right) x_{j} \in V$ for every $x \in F$. If $i \geq q$ and $x \in F$, then $\sum_{j=1}^{\infty} T_{j}^{i} x_{j}-\sum_{j=1}^{\infty} T_{j} x_{j}=$ $\sum_{j=1}^{M}\left(T_{j}^{i}-T_{j}\right) x_{j}+\sum_{j=M+1}^{\infty} T_{j}^{i} x_{j}-\sum_{j=M+1}^{\infty} T_{j} x_{j} \in V+V+V \subset U$.

From Theorems 13 and 15, we have

Theorem 16. (Hahn-Schur) Assume $E$ has signed calF-GHP, $T^{i} \in E^{\beta Y}$ for $i \in N$ and $\lim _{i} T^{i} \cdot x$ exists for every $x \in E$. If for every $j$ there 
exists $T_{j} \in L(X, Y)$ such that $\lim _{i} T_{j}^{i}=T_{j}$ in $L_{c a l F_{j}}(X, Y)$, then $\sum_{j} T_{j}$ is $E$ multiplier convergent and $\lim _{i} \sum_{j=1}^{\infty} T_{j}^{i} x_{j}=\sum_{j=1}^{\infty} T_{j} x_{j}$ uniformly for $x$ belonging to any $F \in$ calF.

From Theorem 16 and Example 4, we obtain a version of the Hahn-Schur Theorem for subseries convergent series; see [Sw2], Chapter 8.1. From Theorem 16 and Example 3, we obtain a version of the Hahn-Schur Theorem for bounded multiplier convergent series, i.e., $l^{\infty}$ multiplier convergent series; see [Sw2] Chapter 8.2. For multiplier spaces with signed-SGHP, see [Sw6] Theorem 8. These scalar results give generalizations of the classical Hahn-Schur Theorem stated above.

We state special cases of Theorem 16 for vector valued multipliers and operator valued series. This gives Hahn-Schur Theorems for operator valued series. From Examples 2 and 3, we have

Corollary 17. Assume $E$ has signed-WGHP (signed-SGHP), $T^{i} \in E^{\beta Y}$ for $i \in N$ and $\lim _{i} T^{i} \cdot x$ exists for each $x \in E$. If for each $j$, there exist $T_{j} \in L(X, Y)$ such that $\lim _{i} T_{j}^{i}=T_{j}$ in $L_{s}(X, Y)\left(L_{b}(X, Y)\right)$, then $\sum_{j} T_{j}$ is $E$ multiplier convergent and $\lim _{i} \sum_{j=1}^{\infty} T_{j}^{i} x_{j}=\sum_{j=1}^{\infty} T_{j} x_{j}$ for each $x \in E$ ( uniformly for $x$ belonging to bounded subsets of $E$ ).

The Hahn-Schur Theorem given in the first statement of Corollary 17 is essentially Stuart's completeness theorem ([St]). Concerning the second statement in Corollary 17, there is an abstract version of the Hahn-Schur Theorem which covers operators and multipliers with the SGHP in [Sw2] Chapter 9.3 and the normed case is discussed in [Sw2] 9.4; see also [Sw3] for the normed case.

Concerning Corollary 8, we obtain

Corollary 18. Assume that $E$ is a complete quasi-normed AK-space with a monotone basis. If $T^{i} \in E^{\beta Y}$ for $i \in N, \lim _{i} T^{i} \cdot x$ exists for each $x \in E$ and there exist $T_{j} \in L(X, Y)$ such that $\lim _{i} T_{j}^{i}=T_{j}$ in $L_{c}(X, Y)$, then $\lim _{i} \sum_{j=1}^{\infty} T_{j}^{i} x_{j}=\sum_{j=1}^{\infty} T_{j} x_{j}$ uniformly for $x$ belonging to compact subsets of $E$.

If the space $X$ is a barrelled locally convex space, the statement in Corollary 18 can be significantly improved since in this case if a sequence of operators with domain $X$ converges in $L_{s}(X, Y)$, then the sequence converges in $L_{c}(X, Y)$, the sequence being equicontinuous ([Sw1]24.11,23.6,[Wi]). 
Corollary 19. Assume that $E$ is a barrelled complete quasi-normed AKspace with a monotone basis. If $T^{i} \in E^{\beta Y}$ for $i \in N, \lim _{i} T^{i} \cdot x$ exists for each $x \in E$ and there exist $T_{j} \in L(X, Y)$ such that $\lim _{i} T_{j}^{i}=T_{j}$ in $L_{s}(X, Y)$, then $\lim _{i} \sum_{j=1}^{\infty} T_{j}^{i} x_{j}=\sum_{j=1}^{\infty} T_{j} x_{j}$ uniformly for $x$ belonging to compact subsets of $E$.

This result is very analogous to the result concerning equicontinuous sequences of operators referenced before Corollary 19 .

We next use the Hahn-Schur Theorem to establish sequential completeness results for $E^{\beta Y}$. Assume that $X, Y$ are locally convex spaces. We denote by $w_{\text {calF }}\left(E^{\beta Y}, E\right)$ the topology on $E^{\beta Y}$ of uniform convergence on the elements of calF. The locally convex topology $w_{c a l F}\left(E^{\beta Y}, E\right)$ is generated by the semi-norms

$$
\pi_{q, F}(T)=\sup \{q(T \cdot x): x \in F\},
$$

where $q$ runs through the continuous semi-norms of $Y$ and $F$ runs through calF.

The pair $(X, Y)$ has the Banach-Steinhaus property (BS property) if whenever $T_{j} \in L(X, Y)$ and $\lim _{j} T_{j} x=T x$ exists for every $x \in X$, then $T \in L(X, Y)$. For example, if $X$ is barrelled, then any pair $(X, Y)$ is has the BS property ([Sw1], [Wi] $)$.

Theorem 20. Assume $E$ has the signed calF-GHP and the pair $(X, Y)$ has the $B S$ property. If $Y$ is sequentially complete, then $w_{c a l F}\left(E^{\beta Y}, E\right)$ is sequentially complete.

Proof: Suppose $\left\{T^{i}\right\}$ is Cauchy in $w_{c a l F}\left(E^{\beta Y}, E\right)$. Then for every $j$ and $z \in X$, the sequence $\left\{T^{i} \cdot\left(e^{j} \otimes z\right)\right\}=\left\{T_{j}^{i} z\right\}$ is Cauchy in $Y$ so $\lim _{i} T_{j}^{i} z=$ $T_{j} z$ exists and $T_{j} \in L(X, Y)$ by the BS property. By Theorem 12 and Proposition 13, $T=\left\{T_{j}\right\} \in E^{\beta Y}$ and $\lim _{i} T^{i} \cdot x=T \cdot x$ for $x \in E$. Given $\epsilon>0, q$ a continuous semi-norm on $Y$ and $F \in c a l F$, there exists $N$ such that $k>l \geq N$ implies $\pi_{q, F}\left(T^{k}-T^{l}\right)<\epsilon$ so $\pi_{q, F}\left(T^{l}-T\right) \leq \epsilon$ for $l \geq N$.

When calF is the family of all finite sets as in Example 2, this is just the sequential completeness result of Stuart ([St]). The results coming from Example 3 (signed-SGHP) and Corollary 8 for uniform convergence on compact sets and Example 9 for uniform convergence over order bounded intervals in solid spaces do not seem to be recorded elsewhere.

Next we consider Orlicz-Pettis theorems for operator valued series. The classical Orlicz-Pettis Theorem asserts that a series in a normed space which 
is subseries convergent in the weak topology is actually subseries convergent in the norm topology of the space $([\mathrm{Or}],[\mathrm{Pe}])$. This theorem has been proven to be very valuable in treating topics in vector measure theory; see [DU], [Ka], [FL] for discussions and historic references. Any theorem which asserts that a series which converges in some topology is convergent in a stronger topology is often referred to as an Orlicz-Pettis Theorem. We consider such theorems for scalar multipliers and operator valued series where the original topology on the space is $L_{s}(X, Y)$. There do not seem to be many Orlicz-Pettis Theorems for the strong operator topology (see [StSw2] Theorem 9 and [Sw5] for examples of such results; however, there are strong results for subseries convergent series in the space of compact operators due to Kalton (see [Sw2] 10.5.6)). Let $\lambda$ be a scalar sequence space which contains the space $c_{00}$ of all sequences which are eventually 0 .

Let calC be the family of all subsets $B$ of $X$ such that when $\left\{x_{k}\right\} \subset B$, there exists a subsequence $\left\{x_{n_{k}}\right\}$ such that $\lim _{k} T x_{n_{k}}$ exists for every $T \in$ $L(X, Y)$. For example, all sequentially compact subsets belong to $\mathrm{calC}$.

Theorem 21. Assume that $\lambda$ has signed calF-GHP and the series $\sum_{j} T_{j}$ is $\lambda$ multiplier convergent in $L_{s}(X, Y)$. If $F \in \mathrm{calF}$ and $B \in \mathrm{calC}$, then the series $\sum_{j=1}^{\infty} t_{j} T_{j} x$ converge uniformly for $t \in F$ and $x \in B$. That is, the series $\sum_{j=1}^{\infty} t_{j} T_{j}$ converge in $L_{\text {calC }}(X, Y)$ uniformly for $t \in F$.

Proof: The proof again uses the Antosik-Mikusinski Matrix Theorem ([St], [Sw2]2.2.4). If the conclusion fails, by Lemma 10 ( the proof ), there exists a symmetric neighborhood $V$ of $0,\left\{t^{k}\right\} \subset F$ and $\left\{x_{k}\right\} \subset B$ and an increasing sequence of intervals $\left\{I_{k}\right\}$ such that

$$
\text { (*) } \sum_{j \in I_{k}} t_{j}^{k} T_{j} x_{k} \notin V \text {. }
$$

By passing to a subsequence if necessary, we may assume that $\lim _{j} T_{i} x_{j}$ exists for every $i$. Consider the matrix

$$
M=\left[m_{i j}\right]=\left[\sum_{l \in I_{j}} t_{l}^{j} T_{l} x_{i}\right] .
$$

We claim that $M$ is a signed calK matrix ([Sw2]2.2.4). First, the columns of $M$ converge by the condition above. Next, given any increasing sequence there is a further subsequence $\left\{n_{j}\right\}$ and a sequence of signs $\left\{s_{j}\right\}$ such that the coordinate sum $u=\sum_{j=1}^{\infty} s_{j} \chi_{I_{n_{j}}} t^{n_{j}} \in \lambda$. Then

$$
\sum_{j=1}^{\infty} s_{j} m_{i n_{j}}=\sum_{j=1}^{\infty} \sum_{l \in I_{n_{j}}} s_{j} t_{l}^{n_{j}} T_{l} x_{i}=\left(\sum_{l=1}^{\infty} u_{l} T_{l}\right) x_{i}
$$


where $\sum_{l=1}^{\infty} u_{l} T_{l}$ is the $L_{s}(X, Y)$ sum of the series. Thus, $\lim _{i} \sum_{j=1}^{\infty} s_{j} m_{i n_{j}}$ exists and $M$ is a signed calK matrix. By the Antosik-Mikusinski Matrix Theorem the diagonal of $M$ converges to 0 . But, this contradicts (*).

Let cal $S C$ be the family of all sequentially compact subsets of $X$. Then as observed above the conclusion of Theorem 21 is valid for the topology $L_{\text {calsC }}(X, Y)$. This observation gives a generalization of Theorem 9 and Corollaries 10 and 11 in [StSw2] where an Orlicz-Pettis Theorem for multiplier spaces with signed-WGHP is given. Theorem 21 also applies to multiplier spaces with signed-SGHP and those in Corollary 8 and Example 9.

Theorem 21 contains a stronger conclusion than most statements of the Orlicz-Pettis theorems in that the series converge uniformly over subsets of the space of multipliers if the multiplier space satisfies a sufficiently strong gliding hump property such as that in Example 3 or Corollary 8 or Example 9. However, see [LS] Theorem 20 for a such a result for multiplier spaces with signed-SGHP.

The proof of Theorem 21 can also be used to obtain Orlicz-Pettis Theorems for subclasses of operators. An operator $T \in L(X, Y)$ is completely continuous if $T$ carries weak convergent sequences in $X$ to convergent sequences in $Y$; denote all such operators by $C C(X, Y)$. For example, any compact operator between normed spaces in completely continuous and the converse holds if $X$ is reflexive or has separable dual ([Sw1]28.1). Note a completely continuous operator carries weak Cauchy sequences in $X$ to Cauchy sequences in $Y$ so if $\left\{x_{k}\right\}$ is weak Cauchy in $X$, then $\lim _{k} T x_{k}$ exists in $Y$ for every $T \in C C(X, Y)$ if $Y$ is sequentially complete. A subset $C$ of $X$ is conditionally weakly compact if every sequence $\left\{x_{k}\right\} \subset C$ has a subsequence $\left\{x_{n_{k}}\right\}$ which is weakly Cauchy ([Di]); denote all such subsets by calWC. The proof of Theorem 21 then gives

Theorem 22. Assume that $\lambda$ has signed calF-GHP and the series $\sum_{j} T_{j}$ is $\lambda$ multiplier convergent in $C C_{s}(X, Y)$ and that $Y$ is sequentially complete. Then for each $F \in$ cal $F$ the series $\sum_{j=1}^{\infty} t_{j} T_{j}$ converge in $C_{\text {calWC }}(X, Y)$ uniformly for $t \in F$.

An operator $T \in L(X, Y)$ is weakly compact if $T$ carries bounded subsets of $X$ into relatively weakly compact subsets of $Y$; denote all such operators by $W(X, Y)$. The space $X$ has the Dunford-Pettis property if every $T \in W(X, Y)$ carries weak Cauchy sequences to convergent sequences (see $[\mathrm{E}]$ for equivalent conditions and examples). As in Theorem 22 we have 
Theorem 23. Assume that $\lambda$ has signed calF-GHP and the series $\sum_{j} T_{j}$ is $\lambda$ multiplier convergent in $W_{s}(X, Y)$ and that $X$ has the Dunford-Pettis property. Then for each $F \in$ cal $F$ the series $\sum_{j=1}^{\infty} t_{j} T_{j}$ converge in $W_{\text {calWC. }}(X, Y)$ uniformly for $t \in F$.

A space $X$ is almost reflexive if every bounded sequence has a weak Cauchy subsequence $([\mathrm{LW}])$. For example, Banach spaces with separable duals, quasi-reflexive Banach spaces and $c_{0}(S)$ are almost reflexive ([LW]). Thus, if $X$ is almost reflexive, then every bounded set belongs to calWC and we have

Theorem 24. Assume that $\lambda$ has signed calF-GHP and the series $\sum_{j} T_{j}$ is $\lambda$ multiplier convergent in $W_{s}(X, Y)$ and that $X$ is almost reflexive and has the Dunford-Pettis property. Then for each $F \in$ calF the series $\sum_{j=1}^{\infty} t_{j} T_{j}$ converge in $W_{b}(X, Y)$ uniformly for $t \in F$.

The results in Theorems 22-24 give generalizations of Theorems 12-14 of [LS] where the case of multipliers with signed-WGHP is treated.

If $Z, Z^{\prime}$ are a pair of vector spaces in duality, let $\sigma\left(Z, Z^{\prime}\right)$ be the weak topology on $Z$. A subset $C \subset Z^{\prime}$ is conditionally $\sigma\left(Z^{\prime}, Z\right)$ sequentially compact if every sequence $\left\{x_{k}^{\prime}\right\} \subset C$ has a subsequence $\left\{x_{n_{k}}^{\prime}\right\}$ such that $\lim _{k} x_{n_{k}}^{\prime}(x)$ exists for every $x \in Z$ ([Di]). The topology on $Z$ of uniform convergence on the family of conditionally $\sigma\left(Z^{\prime}, Z\right)$ sequentially compact subsets of $Z^{\prime}$ is denoted by $\gamma\left(Z, Z^{\prime}\right)$. The proof of Theorem 20 can be repeated to obtain an Orlicz-Pettis Theorem for the topology $\gamma\left(Z, Z^{\prime}\right)$.

Theorem 25. Assume that $\lambda$ has signed calF-GHP and the series $\sum_{j} x_{j}$ is $\lambda$ multiplier convergent in $\sigma\left(Z, Z^{\prime}\right)$. If $F \in$ calF, the series $\sum_{j=1}^{\infty} t_{j} x_{j}$ converge in $\gamma\left(Z, Z^{\prime}\right)$ uniformly for $t \in F$.

Let $\lambda\left(Z, Z^{\prime}\right)$ be the topology on $Z$ of uniform convergence on the $\sigma\left(Z^{\prime}, Z\right)$ compact subsets of $Z^{\prime}$; this topology is stronger than the Mackey topology of $Z$ and can be strictly stronger ([Wi] 9.2.7). The proof of Theorem 2 of [LS] ( see also the proof of Theorem 4.b of [StSw2] ) shows that the statement in Theorem 25 is also valid for the topology $\lambda\left(Z, Z^{\prime}\right)$ and, thus, holds for the Mackey topology. Again the conclusion in Theorem 25 holds uniformly over subsets of the multiplier space, a conclusion not present in many statements of the Orlicz-Pettis Theorem. However, Corollary 21 of [LS] asserts that if $\lambda$ has signed-SGHP, a series $\sum_{j} x_{j}$ which is $\lambda$ multiplier convergent in $\sigma\left(Z, Z^{\prime}\right)$ is such that the series $\sum_{j=1}^{\infty} t_{j} x_{j}$ converges 
uniformly in $\gamma\left(Z, Z^{\prime}\right)$ and $\lambda\left(Z, Z^{\prime}\right)$ for $t=\left\{t_{j}\right\}$ belonging to bounded subsets of $\lambda$, a result which follows directly from Theorem 25 and Example 3. An interesting consequence of an inequality due to McArthur and Retherford ([MR], [Sw2]8.2.1) and the result above is that if $X$ is a sequentially complete locally convex space and $\sum_{j} x_{j}$ is $\sigma\left(X, X^{\prime}\right)$ subseries convergent, then the series $\sum_{j} x_{j}$ is $l^{\infty}$ multiplier convergent and the series $\sum_{j=1}^{\infty} t_{j} x_{j}$ converge uniformly in $\gamma\left(Z, Z^{\prime}\right)$ and $\lambda\left(Z, Z^{\prime}\right)$ for $t=\left\{t_{j}\right\}$ running through the bounded subsets of $l^{\infty}$.

Series which are $c_{0}$ multiplier convergent are used in a number of results pertaining to geometric properties of Banach spaces; for example, a result of Bessaga and Pelczynski asserts that every $c_{0}$ multiplier convergent series in a Banach space $X$ is subseries ( $l^{\infty}$ multiplier) convergent iff $X$ contains no subspace isomorphic to $c_{0}([\mathrm{BP}])$. Theorem 25 and Corollary 8 give an interesting property of $c_{0}$ multiplier convergent series; if a series $\sum_{j} x_{j}$ is $c_{0}$ multiplier convergent with respect to $\sigma\left(Z, Z^{\prime}\right)$, then the series $\sum_{j=1}^{\infty} t_{j} x_{j}$ converge uniformly in $\gamma\left(Z, Z^{\prime}\right)$ and $\lambda\left(Z, Z^{\prime}\right)$ uniformly for $t=\left\{t_{j}\right\}$ running through compact subsets of $c_{0}$. From Corollary 8 similar remarks apply to $c s, b v_{0}$ or $l^{p}(0<p \leq \infty)$ multiplier convergent series.

Similarly, from Example 9 if $\lambda$ is solid and $\sum_{j} x_{j}$ is $\lambda$ multiplier convergent with respect to $\sigma\left(Z, Z^{\prime}\right)$,, then the series $\sum_{j=1}^{\infty} t_{j} x_{j}$ converge uniformly in $\gamma\left(Z, Z^{\prime}\right)$ and $\lambda\left(Z, Z^{\prime}\right)$ uniformly for $t=\left\{t_{j}\right\}$ running through symmetric order intervals in $\lambda$.

These results which contain uniform convergence conclusions for multipliers belonging to subsets of the multiplier space are usually not present in statements of the Orlicz-Pettis Theorem

Using Example 9 we can also obtain some compactness results for sums of $\lambda$ multiplier convergent series. For this we first establish a lemma.

Lemma 26. Let $\sum_{j} x_{j}$ be $\lambda$ multiplier convergent in $X$ and $F \subset \lambda$ be such that $\sum_{j=1}^{\infty} t_{j} x_{j}$ converge uniformly for $t=\left\{t_{j}\right\} \in F$. Let $p$ be the topology of coordinate (pointwise) convergence on $\lambda$. Then the summing operator $S: F \rightarrow X$ defined by $S t=\sum_{j=1}^{\infty} t_{j} x_{j}$ is continuous with respect to $p$ and the topology of $X$.

Proof: Let $t^{\delta}=\left\{t_{j}^{\delta}\right\}$ be a net which converges to $t \in F$ with respect to $p$. Let $U$ be a neighborhood of 0 in $X$ and pick a symmetric neighborhood $V$ such that $V+V+V \subset U$. There exists $n$ such that $\sum_{j=n}^{\infty} s_{j} x_{j} \in V$ for every $s \in F$. There exists $\delta$ such that $\alpha \geq \delta$ implies $\sum_{j<n}\left(t_{j}^{\alpha}-t_{j}\right) x_{j} \in V$. Thus, for $\alpha \geq \delta, S\left(t^{\alpha}\right)-S(t)=\sum_{j<n}\left(t_{j}^{\alpha}-t_{j}\right) x_{j}+\sum_{j=n}^{\infty} t_{j}^{\alpha} x_{j}-\sum_{j=n}^{\infty} t_{j} x_{j} \in$ $V+V+V \subset U$. 
From Theorem 11 and the lemma, we have

Corollary 27. Let $\sum_{j} x_{j}$ be $\lambda$ multiplier convergent in $X$ and $\lambda$ have signed calF GHP. If $F \in$ calF is compact in $p$, then $S=\left\{\sum_{j=1}^{\infty} t_{j} x_{j}: t \in\right.$ $F\}$ is compact in $X$.

From this corollary and Example 9, we have

Corollary 28. If $\lambda$ is solid and $\sum_{j} x_{j}$ is $\lambda$ multiplier convergent in $X$, then for every $t \in \lambda, t \geq 0$, the set $S_{t}=\left\{\sum_{j=1}^{\infty} s_{j} x_{j}:|s| \leq t\right\}$ is compact in $X$.

Proof: The set $[-t, t]=\Pi\left[-t_{j}, t_{j}\right]$ is compact in $p$.

This corollary applies to the AK-spaces $c_{0}, l^{p}(0<p<\infty)$ and also $l^{\infty}, \delta$ and the space $d$ which has no natural vector topology and gives a generalization for a well known result for bounded multiplier convergent series ([Sw2]9.4.1). It follows from the Orlicz-Pettis Theorem 25 that if $\sum_{j} x_{j}$ is $\lambda$ multiplier convergent in $\sigma\left(X, X^{\prime}\right)$ and $\lambda$ is solid, then the set $S_{t}$ is compact in $\lambda\left(X, X^{\prime}\right)$ and $\gamma\left(X, X^{\prime}\right)$ for every $t \in \lambda, t \geq 0$.

P. Antosik has given a very useful criterion for the equality of 2 iterated series when the series have values in a topological group and the series are subseries convergent. Antosik's result was generalized to multiplier convergent series in [Sw7] when the multiplier space $E$ has signed-WGHP. We now give a generalization of the results in [Sw7] to multiplier spaces with signed calF GHP. These generalizations have uniform convergence conclusions.

Theorem 29. Let $E$ have signed calF GHP and $\left\{T_{i j}\right\} \subset L(X, Y)$. Suppose the series $\sum_{j} T_{i j}$ is $\lambda$ multiplier convergent for every $i$ and the iterated series $\sum_{i=1}^{\infty} \sum_{j=1}^{\infty} T_{i j} x_{j}$ converges for every $x=\left\{x_{j}\right\} \in E$. Then the family of iterated series

$$
\left\{\sum_{i=1}^{m} \sum_{j=1}^{\infty} T_{i j} x_{j}: m \in N, x \in F\right\}
$$

converge uniformly for every $F \in$ calF.

Proof: If the conclusion fails, there exists a neighborhood $U$ of 0 in $X$ such that for every $k$ there exist $j_{k}>k, x^{k} \in F$ and $m_{k}$ such that

$$
\sum_{i=1}^{m_{k}} \sum_{j=j_{k}}^{\infty} T_{i j} x_{j}^{k} \notin U .
$$


Pick a balanced neighborhood $V$ such that $V+V \subset U$. There exists $l_{k}>j_{k}$ such that $\sum_{i=1}^{m_{k}} \sum_{j=l_{k}+1}^{\infty} T_{i j} x_{j}^{k} \in V$ so

$$
\text { (*) } \sum_{i=1}^{m_{k}} \sum_{j=j_{k}}^{l_{k}} T_{i j} x_{j}^{k} \notin V .
$$

By the condition $(*)$ for $k=1$, there exist $j_{1}<l_{1}, x^{1} \in F$ and $m_{1}$ such that

$$
\text { (**) } \sum_{i=1}^{m_{1}} \sum_{j=j_{1}}^{l_{1}} T_{i j} x_{j}^{1} \notin V
$$

By Theorem 11, there exists $J_{1}>j_{1}$ such that

$$
(* * *) \quad \sum_{i=1}^{m} \sum_{j=n}^{n+p} T_{i j} x_{j} \in V \text { for } 1 \leq m \leq m_{1}, n>J_{1}, p>0 \text { and } x \in F .
$$

By $(*)$ there exist $l_{2}>j_{2}>J_{1}, m_{2}$ and $x^{2} \in F$ such that $\sum_{i=1}^{m_{2}} \sum_{j=j_{2}}^{l_{2}} T_{i j} x_{j}^{2} \notin$ $V$. By (***) $m_{2}>m_{1}$. Continuing this construction produces increasing sequences $m_{k}, l_{k}, j_{k}$ with $l_{k-1}<j_{k}<l_{k}$ and $x^{k} \in F$ such that

$$
\text { (\#) } \sum_{i=1}^{m_{k}} \sum_{j=j_{k}}^{l_{k}} T_{i j} x_{j}^{k} \notin V \text {. }
$$

Put $I_{k}=\left[j_{k}, l_{k}\right]$ so $\left\{I_{k}\right\}$ is an increasing sequence of intervals.

Define a matrix

$$
M=\left[m_{p q}\right]=\left[\sum_{i=1}^{m_{p}} \sum_{j \in I_{q}} T_{i j} x_{j}^{q}\right]
$$

We claim that $M$ is a signed calK matrix ([Sw2]2.2.4). First note that if $z \in X$ the series $\sum_{i=1}^{\infty} T_{i j} z$ converges for every $j$ by setting $x=e^{j} \otimes z$ in the hypothesis of the theorem. Thus, the columns of $M$ converge. Next, given an increasing sequence of positive integers there is a subsequence $\left\{n_{q}\right\}$ and a sequence of signs $\left\{s_{q}\right\}$ such that the coordinate sum $u=\sum_{q=1}^{\infty} s_{q} \sum_{j \in I_{n_{q}}} x_{j}^{n_{q}} \in \lambda$. Then

$$
\sum_{q=1}^{\infty} s_{q} m_{p n_{q}}=\sum_{q=1}^{\infty} s_{q} \sum_{i=1}^{m_{p}} \sum_{j \in I_{n_{q}}} T_{i j} x_{j}^{n_{q}}=\sum_{i=1}^{m_{p}} \sum_{q=1}^{\infty} s_{q} \sum_{j \in I_{n_{q}}} T_{i j} x_{j}^{n_{q}}=\sum_{i=1}^{m_{p}} \sum_{j=1}^{\infty} T_{i j} u_{j}
$$


so $\lim _{p} \sum_{q=1}^{\infty} s_{q} m_{p n_{q}}=\sum_{i=1}^{\infty} \sum_{j=1}^{\infty} T_{i j} u_{j}$ exists. Hence, $M$ is a signed calK matrix and by the signed version of the Antosik-Mikusinski matrix theorem the diagonal of $M$ converges to 0 . But, this contradicts (***).

From standard limit interchange results, we have the following corollary on the equality of iterated series ([DS]I.7.6,[Ap] 8.20). Here, we denote a double series generated by a double sequence $\left\{x_{i j}\right\}$ in a topological vector space $X$ by $\sum_{i, j} x_{i j}$ and say that a family of double series $\sum_{i, j} x_{i j}(a)$ converge uniformly for $a \in A$ if for every neighborhood of $0, U$, there exists $N$ such that $p, q \geq N$ implies $\sum_{i=p}^{\infty} \sum_{j=q}^{\infty} x_{i j}(a) \in U$ for all $a \in A$.

Corollary 30. Under the hypothesis of Theorem 28 the double series $\sum_{i, j} T_{i j} x_{j}$ converge uniformly for $x \in F$ for every $F \in$ cal $F$. In particular, $\sum_{i=1}^{\infty} \sum_{j=1}^{\infty} T_{i j} x_{j}=$ $\sum_{j=1}^{\infty} \sum_{i=1}^{\infty} T_{i j} x_{j}$ for every $x \in E$.

The case when $E$ has signed-WGHP is the scalar result given in [Sw7]. When $E$ has signed-SGHP the convergence in Theorem 29 and Corollary 30 is uniform over bounded subsets of $E$. Similar remarks apply to the cases of multipliers covered in Corollary 8 and Example 9.

\section{References}

[Ap] T. Apostol, Mathematical Analysis, Addison-Wesley, Reading, 1975.

[BP] C. Bessaga and A. Pelczynski, On Bases and Unconditional Convergence of Series in Banach Spaces, Studia Math., 17 (1958),151-164.

[Bo] J. Boos, Classical and Modern Methods in Summability, Oxford University Press, Oxford ,2000.

[BSS] J. Boos, C. Stuart and C. Swartz, Gliding hump properties of matrix domains, Analysis Math., 30 (2004), 243-257.

[DU] J. Diestel and J. Uhl, Vector Measures, Amer. Math. Soc., Providence, 1977.

[Di] N. Dinculeanu, Weak Compactness and Uniform Convergence of Operators in Spaces of Bochner Integrable Functions, J. Math. Anal. Appl., 109 (1985), 372-387. 
[DS] N. Dunford and J. Schwartz, Linear Operators I, Interscience, N.Y.,1958.

[E] R. E. Edwards, Functional Analysis, Holt-Rinehart-Winston, N.Y., 1965.

[FL] W. Filter and I. Labuda, Essays on the Orlicz-Pettis Theorem I, Real. Anal. Exchange, 16 (1990/91), 393-403.

[Ka] N. Kalton, The Orlicz-Pettis Theorem, Contemporary Math., Amer. Math. Soc., Providence, 1980.

[KG] K.G. Kamthan and M. Gupta, Sequence Spaces and Series, Marcel Dekker, N.Y.,1981.

[Kö] G. Köthe, Topological Vector Spaces, Springer-Verlag, Berlin, 1969.

[LW] E. Lacey and R. Whitley, Conditions under which all the Bounded Linear Maps are Compact, Math. Ann., 158 (1965), 1-5, (1965).

[LS] Li, Ronglu and C. Swartz, An Abstract Orlicz-Pettis Theorem and Applications, Proy. J. Math., 27, pp. 155-169, (2008).

[LWZ] Li, Ronglu and Wang, Fubin and Zhong, S., The strongest intrinsic meaning of sequential-evaluation convergence, Topology and its Appl., 154, pp. 1195-1205, (2007).

[MR] C. W. McArthur and J. Retherford, Some Applications of an Inequality in Locally Convex Spaces, Trans. Amer. Math. Soc., 137, pp. 115-123; (1969).

[MM] G. Metafune and V. B. Moscatelli, On the Space $l^{p+}=\cap_{q>p} l^{q}$, Math. Nachr., 147, pp. 7-12, (1990).

[No] D. Noll, Sequential Completeness and Spaces with the Gliding Hump Property, Manuscripta Math., 66, pp. 237-252, (1990).

[Or] W. Orlicz, Beitrage zur Theorie der Orthogalent wichlungen II, Studia Math., 1, pp. 241-255, (1929).

[Pe] B. J. Pettis, Integration in Vector Spaces, Trans. Amer. Math. Soc. 44, pp. 277-304, (1938).

[St] C. Stuart, Weak Sequential Completeness of $\beta$-duals, Rocky Mountain Math. J., 26, pp. 1559-1568, (1996). 
[StSw1] C. Stuart and C. Swartz, Uniform Convergence in the Dual of a Vector-Valued Sequence Space, Taiwanese J. Math., 7, pp. 665-676, (2003).

[StSw2] C. Stuart and C.Swartz, Generalizations of the Orlicz-Pettis Theorem, Proy. J. Math., 24, pp. 37-48, (2005).

[Sw1] C. Swartz, An Introduction to Functional Analysis, Marcel Dekker, N. Y., (1992).

[Sw2] C. Swartz, Infinite Matrices and the Gliding Hump, World Sci. Publ. Singapore, (1996).

[Sw3] C. Swartz, The Schur and Hahn Theorems for Operator Matrices, Rocky Mountain Math. J., 15, pp. 61-73, (1985).

[Sw4] C. Swartz, A Multiplier Gliding Hump Property for Sequence Spaces, Proy. J. Math., 20, pp. 19-31, (2001).

[Sw5] C. Swartz, Orlicz-Pettis Theorems for Multiplier Convergent Operator Valued Series, Proy. J. Math., 23, pp. 61-72, (2004).

[Sw6] C. Swartz, Uniform Convergence of Multiplier Convergent Series, Proy J. Math., 26, pp. 27-35, (2007).

[Sw7] C. Swartz, Interchanging Orders of Summation for Multiplier Convergent Series, Bol. Soc. Mat. Mexicana (3) 8, pp. 31-35, (2002).

[Wi] . Wilansky, Modern Methods in Topological Vector Spaces, McGrawHill, N. Y., (1978).

[ZLY] hong, S. and Li, Ronglu and Yang, Hong, Summability Results for Matrices of Quasi-Homogeneous Operators, Proy. J. Math., 27, pp. 249-258, (2008).

\section{Charles Swartz}

Department of Mathematics

New Mexico State University

Las Cruces, NM 88003,

U. S. A.

e-mail : cswartz@nmsu.edu 